Bull. Korean Math. Soc. 52 (2015), No. 3, pp. 751-759

http://dx.doi.org/10.4134/BKMS.2015.52.3.751

\title{
NEW CHARACTERIZATIONS OF COMPOSITION OPERATORS BETWEEN BLOCH TYPE SPACES IN THE UNIT BALL
}

\author{
Zhong-Shan FAng And Ze-Hua Zhou
}

\begin{abstract}
In this paper, we give new characterizations of the boundedness and compactness of composition operators $C_{\varphi}$ between Bloch type spaces in the unit ball $\mathbb{B}^{n}$, in terms of the power of the components of $\varphi$, where $\varphi$ is a holomorphic self-map of $\mathbb{B}^{n}$.
\end{abstract}

\section{Introduction}

Let $\mathbb{B}^{n}$ be the unit ball of $\mathbb{C}^{n}$ with boundary $\partial \mathbb{B}^{n}$. The class of all holomorphic functions on $\mathbb{B}^{n}$ will be denoted by $H\left(\mathbb{B}^{n}\right)$. Let $\varphi(z)=\left(\varphi_{1}(z), \ldots, \varphi_{n}(z)\right)$ be a holomorphic self-map of $\mathbb{B}^{n}$. The composition operator is defined as follows:

$$
C_{\varphi}(f)(z)=f(\varphi(z))
$$

for any $f \in H\left(\mathbb{B}^{n}\right)$ and $z \in \mathbb{B}^{n}$.

For any $z=\left(z_{1}, \ldots, z_{n}\right), w=\left(w_{1}, \ldots, w_{n}\right) \in \mathbb{C}^{n}$, the inner product is defined by $\langle z, w\rangle=\sum_{k=1}^{n} z_{k} \bar{w}_{k}$. For $f \in H\left(\mathbb{B}^{n}\right)$, let

$$
\nabla f(z)=\left(\frac{\partial f}{\partial z_{1}}(z), \ldots, \frac{\partial f}{\partial z_{n}}(z)\right)
$$

be the complex gradient of $f$, and

$$
\Re f(z)=\sum_{j=1}^{n} z_{j} \frac{\partial f}{\partial z_{j}}(z)
$$

be the radial derivative of $f$.

Received November 20, 2013; Revised October 26, 2014.

2010 Mathematics Subject Classification. Primary 47B38, 32A18; Secondary 47B33, $32 \mathrm{~A} 10,32 \mathrm{H} 02$

Key words and phrases. composition operator, compactness, Bloch type spaces, unit ball, several complex variables.

The works was supported in part by the National Natural Science Foundation of China (Grant Nos. 11401431; 11371276). The first author was also supported in part by Tianjin City High School Science and Technology Fund Planning Project (Grant No. 20141002). 
Given $\varphi(z)=\left(\varphi_{1}(z), \ldots, \varphi_{n}(z)\right)$ a holomorphic self-map of $\mathbb{B}^{n}$, the symbol $\varphi^{\prime}(z) z$ is denoted by

$$
\varphi^{\prime}(z) z=\left(\Re \varphi_{1}(z), \ldots, \Re \varphi_{n}(z)\right) .
$$

For any $0<p<\infty$, we define the Bloch type space $\mathcal{B}^{p}$ as the space of holomorphic functions such that

$$
\|f\|_{p}=|f(0)|+\sup \left\{\left(1-|z|^{2}\right)^{p}|\Re f(z)|: z \in \mathbb{B}^{n}\right\}<\infty .
$$

It is important to provide function-theoretic descriptions of when $\varphi$ induces a bounded or compact composition operator on various function spaces. For general references on the theory of composition operators, we refer the interested readers to the books $[1,14,25]$. Recently there has been a great interest in studying composition operators between Bloch type spaces. For example, see $[2,5,11,13,15,20,21,22,23,24]$. So far there are still many unsolved problems that are the interest of numerous mathematicians. In [18], Wulan, Zheng and Zhu obtained a new result about the compactness of the composition operator on the Bloch space in the unit disk. Recently, interest has arisen to characterize the boundedness and compactness of (weighted) composition operators $(u) C_{\varphi}$ on Bloch type spaces in terms of the $n$-th power of the analytic self-map $\varphi$ on the unit disc $\mathbb{D}$, such as $[4,7,8,9,10,12,17,19]$.

For the higher dimensional case, in 2012, the authors in [6] generalize Zhao's results in [19] to the polydisk. Unlike the case of composition operators on the unit disk, the essential norms are different for the cases $p \in(0,1)$ and $p \geq 1$. Dai in [3] gave several new necessary and sufficient conditions for the compactness of the composition operator on the Bloch space in the unit ball. Along with this line, this paper will consider the new characterizations of the boundedness and compactness of composition operators between Bloch type spaces in the unit ball.

\section{Some lemmas}

In this section, we present some lemmas which will be used in the proofs of our main results in the next section.

The proof of the following lemma can be found in Lemma 2.2 in [19].

Lemma 2.1. Let $p>0, m \in \mathbb{N}$ and $0 \leq x \leq 1$. And let $H_{m, p}(x)=x^{m-1}(1-$ $\left.x^{2}\right)^{p}, r_{m}=\left(\frac{m-1}{m-1+2 p}\right)^{1 / 2} \quad(m \geq 2)$, and $r_{m}=0$ for $m=1$. Then for $m \geq 1$, $H_{m, p}$ has the following properties.

(i) $\max _{0 \leq x \leq 1} H_{m, p}(x)=H_{m, p}\left(r_{m}\right)= \begin{cases}1, & m=1 ; \\ \left(\frac{2 p}{m-1+2 p}\right)^{p}\left(\frac{m-1}{m-1+2 p}\right)^{(m-1) / 2}, & m \geq 2,\end{cases}$ and

$$
\lim _{m \rightarrow \infty} m^{p} \max _{x \in[0,1]} H_{m, p}(x)=\left(\frac{2 p}{e}\right)^{p} .
$$


(ii) $H_{m, p}(x)$ is decreasing on $\left[r_{m}, r_{m+1}\right]$, and

$$
\lim _{m \rightarrow \infty} m^{p} \min _{x \in\left[r_{m}, r_{m+1}\right]} H_{m, p}(x)=\left(\frac{2 p}{e}\right)^{p} .
$$

Combining Lemma 3.1 with Theorem 6.1 in [2], we obtain the lemma below.

Lemma 2.2. Let $p>1 / 2, q>0$. Suppose that $\varphi=\left(\varphi_{1}, \ldots, \varphi_{n}\right)$ is a holomorphic self-map of $\mathbb{B}^{n}$. Then $C_{\varphi}: \mathcal{B}^{p} \rightarrow \mathcal{B}^{q}$ is bounded if and only if

$$
\sup _{z \in \mathbb{B}^{n}} \frac{\left(1-|z|^{2}\right)^{q}\left\{\left(1-|\varphi(z)|^{2}\right)\left|\varphi^{\prime}(z) z\right|^{2}+\left|\left\langle\varphi^{\prime}(z) z, \varphi(z)\right\rangle\right|^{2}\right\}^{1 / 2}}{\left(1-|\varphi(z)|^{2}\right)^{p}}<\infty .
$$

Due to Lemma 3.1 and Theorem 7.4 in [2], the next lemma follows.

Lemma 2.3. Let $p, q>0$. Suppose that $\varphi=\left(\varphi_{1}, \ldots, \varphi_{n}\right)$ is a holomorphic self-map of $\mathbb{B}^{n}$, and $C_{\varphi}: \mathcal{B}^{p} \rightarrow \mathcal{B}^{q}$ is bounded. Then the following statements are true:

(i) If $0<p<1 / 2$, then $C_{\varphi}: \mathcal{B}^{p} \rightarrow \mathcal{B}^{q}$ is compact if and only if

$$
\lim _{|\varphi(z)| \rightarrow 1} \frac{\left(1-|z|^{2}\right)^{q}\left|\left\langle\varphi^{\prime}(z) z, \varphi(z)\right\rangle\right|}{\left(1-|\varphi(z)|^{2}\right)^{p}}=0 ;
$$

(ii) If $p>1 / 2$, then $C_{\varphi}: \mathcal{B}^{p} \rightarrow \mathcal{B}^{q}$ is compact if and only if

$$
\lim _{|\varphi(z)| \rightarrow 1} \frac{\left(1-|z|^{2}\right)^{q}\left\{\left(1-|\varphi(z)|^{2}\right)\left|\varphi^{\prime}(z) z\right|^{2}+\left|\left\langle\varphi^{\prime}(z) z, \varphi(z)\right\rangle\right|^{2}\right\}^{1 / 2}}{\left(1-|\varphi(z)|^{2}\right)^{p}}=0 \text {. }
$$

Lemma 2.4. Let $p>0, m \in \mathbb{N}, \xi \in \partial \mathbb{B}^{n}$. Then

$$
\lim _{m \rightarrow \infty} \sup _{\xi \in \partial \mathbb{B}^{n}} m^{p-1}\left\|\langle z, \xi\rangle^{m}\right\|_{p} \leq\left(\frac{2 p}{e}\right)^{p} .
$$

Proof. Note that for any $\xi \in \partial \mathbb{B}^{n}$,

$$
\begin{aligned}
\sup _{\xi \in \partial \mathbb{B}^{n}} m^{p-1}\left\|\langle z, \xi\rangle^{m}\right\|_{p} & =\sup _{\xi \in \partial \mathbb{B}^{n}} m^{p-1} \sup _{z \in \mathbb{B}^{n}}\left(1-|z|^{2}\right)^{p}\left|\Re\left(\langle z, \xi\rangle^{m}\right)\right| \\
& =\sup _{\xi \in \partial \mathbb{B}^{n}} m^{p-1} \sup _{z \in \mathbb{B}^{n}} m\left(1-|z|^{2}\right)^{p}\left|\langle z, \xi\rangle^{m-1}\right| \cdot|\langle z, \xi\rangle| \\
& \leq \sup _{z \in \mathbb{B}^{n}} m^{p}\left(1-|z|^{2}\right)^{p}|z|^{m-1} .
\end{aligned}
$$

Using (i) of Lemma 2.1, the lemma follows immediately.

The following lemma is the crucial criterion for the compactness of $C_{\varphi}$, whose proof is similar as that of Proposition 3.11 in [1].

Lemma 2.5. Assume that $\varphi$ is a holomorphic self-map of $\mathbb{B}^{n}$. Then $C_{\varphi}$ : $\mathcal{B}^{p} \rightarrow \mathcal{B}^{q}$ is compact if and only if $C_{\varphi}$ is bounded and for any bounded sequence $\left\{f_{m}\right\}_{m \in \mathbb{N}}$ in $\mathcal{B}^{p}$ which converges to zero uniformly on compact subsets of $\mathbb{B}^{n}$, we have

as $m \rightarrow \infty$.

$$
\left\|C_{\varphi} f_{m}\right\|_{q} \rightarrow 0
$$




\section{Main theorems}

In this section, we will characterize the boundedness and compactness of composition operators between Bloch type spaces in terms of the power of the components of $\varphi$.

Theorem 3.1. Let $p, q>0$. Suppose that $\varphi=\left(\varphi_{1}, \ldots, \varphi_{n}\right)$ is a holomorphic self-map of $\mathbb{B}^{n}$. Then $C_{\varphi}: \mathcal{B}^{p} \rightarrow \mathcal{B}^{q}$ is bounded if and only if

$$
\sup _{m \in \mathbb{N}} \sup _{\xi \in \partial \mathbb{B}^{n}} m^{p-1}\left\|\langle\varphi, \xi\rangle^{m}\right\|_{q}<\infty .
$$

Proof. Suppose that $C_{\varphi}$ is bounded. And for $m \in \mathbb{N}, \xi \in \partial \mathbb{B}^{n}$, consider the test functions $g_{m, \xi}(z)=\frac{\langle z, \xi\rangle^{m}}{\left\|\langle z, \xi\rangle^{m}\right\|_{p}}$.

By Lemma 2.4, there is a constant $C>0$, independent of $m$ and $\xi$, such that $\left\|\langle z, \xi\rangle^{m}\right\|_{p} \leq C m^{1-p}$, therefore,

$$
\infty>\left\|C_{\varphi}\right\| \geq\left\|C_{\varphi} g_{m, \xi}\right\|_{q}=\frac{\left\|\langle\varphi, \xi\rangle^{m}\right\|_{q}}{\left\|\langle z, \xi\rangle^{m}\right\|_{p}} \geq \frac{1}{C} m^{p-1}\left\|\langle\varphi, \xi\rangle^{m}\right\|_{q} .
$$

Thus

$$
\sup _{m \in \mathbb{N}} \sup _{\xi \in \partial \mathbb{B}^{n}} m^{p-1}\left\|\langle\varphi, \xi\rangle^{m}\right\|_{q} \leq C\left\|C_{\varphi}\right\|<\infty .
$$

Conversely, for any integer $m \geq 1$, let

$$
A_{m}=\left\{z \in \mathbb{B}^{n}: r_{m} \leq|\varphi(z)| \leq r_{m+1}\right\}
$$

where $r_{m}$ is defined in Lemma 2.1. Let $k$ be the largest positive integer such that $A_{m} \neq \emptyset(k$ could be $\infty)$. Thus $\mathbb{B}^{n}=\bigcup_{m=1}^{k} A_{m}$. From (ii) of Lemma 2.1, we know that, there exists a large enough integer $N$ and a constant $\delta_{1}>0$, such that

$$
\min _{z \in A_{m}} m^{p}|\varphi(z)|^{m-1}\left(1-|\varphi(z)|^{2}\right)^{p} \geq \delta_{1}
$$

whenever $m>N$. Noticing that also by Lemma 2.1,

$$
\min _{z \in A_{m}} m^{p}|\varphi(z)|^{m-1}\left(1-|\varphi(z)|^{2}\right)^{p} \geq m^{p} H_{m, p}\left(r_{m+1}\right),
$$

set $\delta_{2}=\min _{1 \leq m \leq N}\left\{m^{p} H_{m, p}\left(r_{m+1}\right)\right\}$ and denote $\delta=\min \left\{\delta_{1}, \delta_{2}\right\}$. Therefore, there exists a constant $\delta>0$, independent of $\mathrm{m}$, such that

$$
\min _{z \in A_{m}} m^{p}|\varphi(z)|^{m-1}\left(1-|\varphi(z)|^{2}\right)^{p} \geq \delta .
$$

Hence, we have

$$
\begin{aligned}
& \sup _{z \in \mathbb{B}^{n}} \frac{\left(1-|z|^{2}\right)^{q}\left|\left\langle\varphi^{\prime}(z) z, \varphi(z)\right\rangle\right|}{\left(1-|\varphi(z)|^{2}\right)^{p}} \\
\leq & \sup _{m \geq 1} \sup _{z \in A_{m}} \frac{m^{p}|\varphi(z)|^{m-1}\left(1-|z|^{2}\right)^{q}\left|\left\langle\varphi^{\prime}(z) z, \varphi(z)\right\rangle\right|}{m^{p}|\varphi(z)|^{m-1}\left(1-|\varphi(z)|^{2}\right)^{p}} \\
\leq & \frac{1}{\delta} \sup _{m \geq 1} \sup _{z \in A_{m}} m^{p}|\varphi(z)|^{m-1}\left(1-|z|^{2}\right)^{q}\left|\left\langle\varphi^{\prime}(z) z, \varphi(z)\right\rangle\right|
\end{aligned}
$$




$$
\begin{aligned}
& \leq \frac{1}{\delta} \sup _{m \geq 1} \sup _{z \in A_{m}} m^{p}|\varphi(z)|^{m-2}\left(1-|z|^{2}\right)^{q}\left|\left\langle\varphi^{\prime}(z) z, \varphi(z)\right\rangle\right| \\
& =\frac{1}{\delta} \sup _{m \geq 1} \sup _{z \in A_{m}} m^{p}\left(1-|z|^{2}\right)^{q}\left|\left\langle\varphi(z), \frac{\varphi(z)}{|\varphi(z)|}\right\rangle^{m-1}\right| \cdot\left|\left\langle\varphi^{\prime}(z) z, \frac{\varphi(z)}{|\varphi(z)|}\right\rangle\right| \\
& \leq \frac{1}{\delta} \sup _{m \geq 1} \sup _{\xi \in \partial \mathbb{B}^{n}} m^{p-1} \sup _{z \in \mathbb{B}^{n}} m\left(1-|z|^{2}\right)^{q}\left|\langle\varphi(z), \xi\rangle^{m-1}\right| \cdot\left|\left\langle\varphi^{\prime}(z) z, \xi\right\rangle\right| \\
& \leq \frac{1}{\delta} \sup _{m \geq 1} \sup _{\xi \in \partial \mathbb{B}^{n}} m^{p-1} \sup _{z \in \mathbb{B}^{n}}\left(1-|z|^{2}\right)^{q}\left|\Re\left(\langle\varphi(z), \xi\rangle^{m}\right)\right| \\
& \leq \frac{1}{\delta} \sup _{m \geq 1} \sup _{\xi \in \partial \mathbb{B}^{n}} m^{p-1}\left\|\langle\varphi, \xi\rangle^{m}\right\|_{q}<\infty
\end{aligned}
$$

Case 1. Whenever $|\varphi(z)| \leq 1 / 2$, for $i \in\{1,2, \ldots, n\}$, set $\xi=e_{i}=$ $(0, \ldots, 1, \ldots, 0)$, where the $i$-th component is 1 , otherwise 0 . Then by the assumption, $\left\|\left\langle\varphi(z), e_{i}\right\rangle\right\|_{q}=\left\|\varphi_{i}\right\|_{q}=|\varphi(0)|+\sup _{z \in \mathbb{B}^{n}}\left(1-|z|^{2}\right)^{q}\left|\Re \varphi_{i}(z)\right|<\infty$. Therefore,

$$
\begin{aligned}
& \frac{\left(1-|z|^{2}\right)^{q}\left\{\left(1-|\varphi(z)|^{2}\right)\left|\varphi^{\prime}(z) z\right|^{2}\right\}^{1 / 2}}{\left(1-|\varphi(z)|^{2}\right)^{p}} \\
\leq & \left(\frac{4}{3}\right)^{p}\left(1-|z|^{2}\right)^{q}\left\{\left|\varphi^{\prime}(z) z\right|^{2}\right\}^{1 / 2} \\
\leq & \left(\frac{4}{3}\right)^{p}\left(1-|z|^{2}\right)^{q}\left\{\left|\Re \varphi_{1}(z)\right|^{2}+\left|\Re \varphi_{2}(z)\right|^{2} \cdots+\left|\Re \varphi_{n}(z)\right|^{2}\right\}^{1 / 2}<\infty .
\end{aligned}
$$

Case 2. If $|\varphi(z)|>1 / 2$, by the projection theorem, there exists $\eta(z) \in \partial \mathbb{B}^{n}$, such that $\langle\varphi(z), \eta(z)\rangle=0$ and

$$
\varphi^{\prime}(z) z=u \varphi(z)+v \eta(z)
$$

where

Therefore,

$$
u=\frac{\left\langle\varphi^{\prime}(z) z, \varphi(z)\right\rangle}{|\varphi(z)|}, v=\left\langle\varphi^{\prime}(z) z, \eta(z)\right\rangle
$$

$$
\begin{aligned}
& \frac{\left(1-|z|^{2}\right)^{q}\left\{\left(1-|\varphi(z)|^{2}\right)\left|\varphi^{\prime}(z) z\right|^{2}\right\}^{1 / 2}}{\left(1-|\varphi(z)|^{2}\right)^{p}} \\
= & \frac{\left(1-|z|^{2}\right)^{q}\left\{\left(1-|\varphi(z)|^{2}\right)\left(|u|^{2}+|v|^{2}\right)\right\}^{1 / 2}}{\left(1-|\varphi(z)|^{2}\right)^{p}} \\
\leq & \frac{2\left(1-|z|^{2}\right)^{q}|u|\left(1-|\varphi(z)|^{2}\right)^{1 / 2}}{\left(1-|\varphi(z)|^{2}\right)^{p}}+\frac{2\left(1-|z|^{2}\right)^{q}|v|\left(1-|\varphi(z)|^{2}\right)^{1 / 2}}{\left(1-|\varphi(z)|^{2}\right)^{p}} \\
= & I_{1}+I_{2} .
\end{aligned}
$$

Obviously, by (3.1), we have $I_{1}<\infty$. Now let $\zeta=\varphi(z)+\sqrt{1-|\varphi(z)|^{2}} \eta(z)$, then $|\zeta|=1$, and

$$
\langle\varphi(z), \zeta\rangle=|\varphi(z)|^{2},\left\langle\varphi^{\prime}(z) z, \zeta\right\rangle=\left\langle\varphi^{\prime}(z) z, \varphi(z)\right\rangle+\sqrt{1-|\varphi(z)|^{2}} v .
$$


Then we obtain that

$$
\begin{aligned}
& \sup _{m \geq 1} m^{p-1}\left\|\langle\varphi, \zeta\rangle^{m}\right\|_{q} \\
\geq & \sup _{m \geq 1} m^{p-1} \sup _{z \in \mathbb{B}^{n}} m\left(1-|z|^{2}\right)^{q}\left|\langle\varphi(z), \zeta\rangle^{m-1}\right| \cdot\left|\left\langle\varphi^{\prime}(z) z, \zeta\right\rangle\right| \\
= & \sup _{m \geq 1} m^{p-1} \sup _{z \in \mathbb{B}^{n}} m\left(1-|z|^{2}\right)^{q}|\varphi(z)|^{2 m-2} \cdot\left|\left\langle\varphi^{\prime}(z) z, \zeta\right\rangle\right| .
\end{aligned}
$$

Similar arguments as for (3.1), it is easy to show

$$
\sup _{z \in \mathbb{B}^{n}} \frac{\left(1-|z|^{2}\right)^{q}\left|\left\langle\varphi^{\prime}(z) z, \zeta\right\rangle\right|}{\left(1-|\varphi(z)|^{2}\right)^{p}}<\infty .
$$

Note that

$$
|v| \sqrt{1-|\varphi(z)|^{2}} \leq\left|\left\langle\varphi^{\prime}(z) z, \zeta\right\rangle\right|+\left\langle\varphi^{\prime}(z) z, \varphi(z)\right\rangle,
$$

so $I_{2}<\infty$. Combining the two cases above, we have actually show that

$$
\sup _{z \in \mathbb{B}^{n}} \frac{\left(1-|z|^{2}\right)^{q}\left\{\left(1-|\varphi(z)|^{2}\right)\left|\varphi^{\prime}(z) z\right|^{2}\right\}^{1 / 2}}{\left(1-|\varphi(z)|^{2}\right)^{p}}<\infty .
$$

The theorem follows by Lemma 2.2 .

Theorem 3.2. Let $p, q>0, p \neq 1 / 2$. Suppose that $\varphi=\left(\varphi_{1}, \ldots, \varphi_{n}\right)$ is a holomorphic self-map of $\mathbb{B}^{n}$. Then $C_{\varphi}: \mathcal{B}^{p} \rightarrow \mathcal{B}^{q}$ is compact if and only if

$$
\lim _{m \rightarrow \infty} \sup _{\xi \in \partial \mathbb{B}^{n}} m^{p-1}\left\|\langle\varphi, \xi\rangle^{m}\right\|_{q}=0 .
$$

Proof. We show the necessity first. For $m \in \mathbb{N}$ and $\xi \in \partial \mathbb{B}^{n}$, consider the test functions $g_{m, \xi}(z)=\frac{\langle z, \xi\rangle^{m}}{\left\|\langle z, \xi\rangle^{m}\right\|_{p}}$, then it is easy to see that $\left\|g_{m, \xi}\right\|_{p}=1$ and $g_{m, \xi}(z) \rightarrow 0$ uniformly on compact subsets of $\mathbb{B}^{n}$, as $m \rightarrow \infty$. If $C_{\varphi}: \mathcal{B}^{p} \rightarrow \mathcal{B}^{q}$ is compact, by Lemmas 2.4 and 2.5, we have

$$
\begin{aligned}
0 & =\lim _{m \rightarrow \infty}\left\|C_{\varphi} g_{m, \xi}\right\|_{q}=\lim _{m \rightarrow \infty} \frac{\left\|\langle\varphi, \xi\rangle^{m}\right\|_{q}}{\left\|\langle z, \xi\rangle^{m}\right\|_{p}} \\
& =\lim _{m \rightarrow \infty} \frac{m^{p-1}\left\|\langle\varphi, \xi\rangle^{m}\right\|_{q}}{m^{p-1}\left\|\langle z, \xi\rangle^{m}\right\|_{p}} \\
& \geq\left(\frac{e}{2 p}\right)^{p} \lim _{m \rightarrow \infty} m^{p-1}\left\|\langle\varphi, \xi\rangle^{m}\right\|_{q} .
\end{aligned}
$$

Since $\xi$ is arbitrary, the necessity follows.

Now we turn to prove the sufficiency. Assume that $m^{p-1}\left\|\langle\varphi, \xi\rangle^{m}\right\|_{q} \rightarrow 0$ uniformly on $\partial \mathbb{B}^{n}$. For each integer $m \geq 1$, let $A_{m}$ be as in Theorem 3.1, similar as discussed above, then we have

$$
\begin{aligned}
0 & =\lim _{m \rightarrow \infty} \sup _{\xi \in \partial \mathbb{B}^{n}} m^{p-1}\left\|\langle\varphi, \xi\rangle^{m}\right\|_{q} \\
& \geq \lim _{m \rightarrow \infty} \sup _{\xi \in \partial \mathbb{B}^{n}} m^{p-1} \sup _{z \in \mathbb{B}^{n}}\left(1-|z|^{2}\right)^{q}\left|\Re\left(\langle\varphi(z), \xi\rangle^{m}\right)\right|
\end{aligned}
$$




$$
\begin{aligned}
& =\lim _{m \rightarrow \infty} \sup _{\xi \in \partial \mathbb{B}^{n}} \sup _{z \in \mathbb{B}^{n}} m^{p}\left(1-|z|^{2}\right)^{q}\left|\langle\varphi(z), \xi\rangle^{m-1}\right| \cdot\left|\left\langle\varphi^{\prime}(z) z, \xi\right\rangle\right| \\
& \geq \lim _{m \rightarrow \infty} \sup _{z \in A_{m}} m^{p}\left(1-|z|^{2}\right)^{q}\left|\left\langle\varphi(z), \frac{\varphi(z)}{|\varphi(z)|}\right\rangle^{m-1}\right| \cdot\left|\left\langle\varphi^{\prime}(z) z, \frac{\varphi(z)}{|\varphi(z)|}\right\rangle\right| \\
& =\lim _{m \rightarrow \infty} \sup _{z \in A_{m}} m^{p}\left(1-|z|^{2}\right)^{q}|\varphi(z)|^{m-2}\left|\left\langle\varphi^{\prime}(z) z, \varphi(z)\right\rangle\right| \\
& \geq \lim _{m \rightarrow \infty} \sup _{z \in A_{m}} \frac{\left(1-|z|^{2}\right)^{q} m^{p}\left(1-|\varphi(z)|^{2}\right)^{p}|\varphi(z)|^{m-1}}{\left(1-|\varphi(z)|^{2}\right)^{p}}\left|\left\langle\varphi^{\prime}(z) z, \varphi(z)\right\rangle\right| \\
& \geq\left(\frac{2 p}{e}\right)^{p} \lim _{m \rightarrow \infty} \sup _{z \in A_{m}} \frac{\left(1-|z|^{2}\right)^{q}\left|\left\langle\varphi^{\prime}(z) z, \varphi(z)\right\rangle\right|}{\left(1-|\varphi(z)|^{2}\right)^{p}} \\
& \geq\left(\frac{2 p}{e}\right)^{p} \lim _{|z| \rightarrow 1} \frac{\left(1-|z|^{2}\right)^{q}\left|\left\langle\varphi^{\prime}(z) z, \varphi(z)\right\rangle\right|}{\left(1-|\varphi(z)|^{2}\right)^{p}} . \\
& 0<p<1 / 2, \text { the theorem follows by (i) of Lemma 2.3. }
\end{aligned}
$$

To prove the theorem for the case $p>1 / 2$, by (ii) of Lemma 2.3 , we just need to show that

$$
\lim _{|\varphi(z)| \rightarrow 1} \frac{\left(1-|z|^{2}\right)^{q}\left\{\left(1-|\varphi(z)|^{2}\right)\left|\varphi^{\prime}(z) z\right|^{2}\right\}^{1 / 2}}{\left(1-|\varphi(z)|^{2}\right)^{p}}=0 .
$$

The same as proof in (3.2), we have

$$
\frac{\left(1-|z|^{2}\right)^{q}\left\{\left(1-|\varphi(z)|^{2}\right)\left|\varphi^{\prime}(z) z\right|^{2}\right\}^{1 / 2}}{\left(1-|\varphi(z)|^{2}\right)^{p}} \leq I_{1}+I_{2}
$$

where $I_{1}, I_{2}$ are defined the same as in the previous theorem. Next we will show that $I_{1} \rightarrow 0, I_{2} \rightarrow 0$ as $|\varphi(z)| \rightarrow 1$. From (3.4), it is obvious that $I_{1} \rightarrow 0$, as $|\varphi(z)| \rightarrow 1$. Now let $\zeta=\varphi(z)+\sqrt{1-|\varphi(z)|^{2}} \eta(z)$, where $\eta(z)$ is defined as in Theorem 3.1. Noticing that (3.1) and Lemma 2.1, similar arguments show that

$$
\begin{aligned}
& \lim _{m \rightarrow \infty} m^{p-1}\left\|\langle\varphi, \zeta\rangle^{m}\right\|_{q} \\
\geq & \lim _{m \rightarrow \infty} m^{p-1} \sup _{z \in \mathbb{B}^{n}} m\left(1-|z|^{2}\right)^{q}\left|\langle\varphi(z), \zeta\rangle^{m-1}\right| \cdot\left|\left\langle\varphi^{\prime}(z) z, \zeta\right\rangle\right| \\
= & \lim _{m \rightarrow \infty} m^{p-1} \sup _{z \in \mathbb{B}^{n}} m\left(1-|z|^{2}\right)^{q}|\varphi(z)|^{2 m-2} \cdot\left|\left\langle\varphi^{\prime}(z) z, \zeta\right\rangle\right|
\end{aligned}
$$

and

$$
\lim _{|\varphi(z)| \rightarrow 1} \frac{\left(1-|z|^{2}\right)^{q}\left|\left\langle\varphi^{\prime}(z) z, \zeta\right\rangle\right|}{\left(1-|\varphi(z)|^{2}\right)^{p}}=0 .
$$

Combining (3.4) with (3.5), again using (3.3), we obtain that $I_{2} \rightarrow 0$ as $|\varphi(z)| \rightarrow 1$. This completes the proof of the theorem.

For technical reasons we can't deal with the case $p=1 / 2$, so we pose the following question.

Question. What about the case $p=1 / 2$, is it compatible with Theorem 3.2? 
Acknowledgments. We would like to thank the referee for useful comments and suggestions which improved the presentation of this paper.

\section{References}

1] C. C. Cowen and B. D. MacCluer, Composition Operators on Spaces of Analytic Functions, CRC Press, Boca Raton, 1995.

[2] H. Chen and P. Gauthier, Composition operators on $\mu$-Bloch spaces, Canad. J. Math. 61 (2009), no. 1, 50-75.

[3] J. Dai, Compact composition operators on the Bloch space of the unit ball, J. Math. Anal. Appl. 386 (2012), no. 1, 294-299.

[4] K. Esmaeili and M. Lindström, Weighted composition operators between Zygmund type spaces and their essential norms, Integral Equations Operator Theory $\mathbf{7 5}$ (2013), no. 4, 473-490.

[5] Z. S. Fang, Compact composition operators between Bloch type spaces in the polydisk, Abstr. Appl. Anal. 2012 (2012), Article ID 732709, 7 pp.

[6] Z. S. Fang and Z. H. Zhou, Essential norms of composition operators between Bloch type spaces in the polydisk, Arch. Math. (Basel) 99 (2012), no. 6, 547-556.

[7] O. Hyvärinen, M. Kemppainen, M. Lindström, A. Rautio, and E. Saukko, The essential norm of weighted composition operators on weighted Banach spaces of analytic functions, Integral Equations Operator Theory 72 (2012), no. 2, 151-157.

[8] O. Hyvärinen and M. Lindström, Estimates of essential norms of weighted composition operators between Bloch-type spaces, J. Math. Anal. Appl. 393 (2012), no. 1, 38-44.

[9] Y. X. Liang and Z. H. Zhou, Essential norm of the product of differentiation and composition operators between Bloch-type spaces, Arch. Math. (Basel) 100 (2013), no. 4, $347-360$.

[10] New estimate of essential norm of composition followed by differentiation between Bloch-type spces, Banach J. Math. Anal. 7 (2013), 160-172.

[11] K. Madigan and A. Matheson, Compact composition operators on the Bloch space, Trans. Amer. Math. Soc. 347 (1995), no. 7, 2679-2687.

[12] J. Manhas and R. Zhao, New estimates of essential norms of weighted composition operators between Bloch type spaces, J. Math. Anal. Appl. 389 (2012), no. 1, 32-47.

[13] J. H. Shapiro, The essential norm of a composition operator, Ann. Math. 125 (1987), no. $2,375-404$.

[14] Composition Operators and Classical Function Theory, Springer-Verlag, New York, 1993.

[15] S. Stević, On new Bloch-type spaces, Appl. Math. Comput. 215 (2009), no. 2, 841-849.

[16] S. Stević, R. Y. Chen, and Z. H. Zhou, Weighted composition operators between Blochtype spaces in the polydisk, Sbornik Mathematics 201 (2010), no. 2, 289-319.

[17] Y. Wu and H. Wulan, Products of differentiation and composition operators on the Bloch space, Collect. Math. 63 (2012), no. 1, 93-107.

[18] H. Wulan, D. Zheng, and K. Zhu, Compact composition operators on BMO and the Bloch space, Proc. Amer. Math. Soc. 137 (2009), no. 11, 3861-3868.

[19] R. Zhao, Essential norms of composition operators between Bloch type spaces, Proc. Amer. Math. Soc. 138 (2010), no. 7, 2537-2546.

[20] Z. H. Zhou, Y. X. Liang, and X. T. Dong, Weighted composition operator between weighted-type space and Hardy space on the unit ball, Ann. Polon. Math. 104 (2012), no. 3, 309-319.

[21] Z. H. Zhou and J. H. Shi, Compact composition operators on the Bloch space in the polydisk, Sci. China Ser. A 44 (2001), no. 3, 286-291.

[22] Composition operators on the Bloch space in the polydisk, Complex Variables 46 (2001), no. 1, 73-88. 
[23] - Compactness of composition operators on the Bloch space in classical bounded symmetric domains, Michigan Math. J. 50 (2002), no. 2, 381-405.

[24] H. G. Zeng and Z. H. Zhou, Essential norm estimate of a composition operator between Bloch-type spaces in the unit ball, Rocky Mountain J. Math. 42 (2012), no. 3, 1049-1071.

[25] K. Zhu, Operator Theory in Function Spaces, Marcel Dekker, New York, 1990.

ZHONG-SHAN FANG

Department of Mathematics

Tianjin Polytechnic University

Tianjin, 300387, P. R. China

E-mail address: fangzhongshan@aliyun.com

Ze-Hua Zhou

Department of Mathematics

Tianjin University

Tianjin, 300072, P. R. ChinA

E-mail address: zehuazhoumath@aliyun.com; zhzhou@tju.edu.cn 